\title{
JHore LHomme
}

Revue française d'anthropologie

Olivier Leservoisier, ed., Terrains ethnographiques et hiérarchies sociales. Retour réflexif sur la situation d'enquête

Paris, Karthala, 2005, 327 p., bibl., index (« Hommes et sociétés »)

Samuel Lézé

\section{OpenEdition}

Journals

Édition électronique

URL : http://journals.openedition.org//homme/9651

DOI : 10.4000/lhomme.9651

ISSN : 1953-8103

Éditeur

Éditions de l'EHESS

Édition imprimée

Date de publication : 1 septembre 2007

Pagination : 199-201

ISSN : 0439-4216

Référence électronique

Samuel Lézé, « Olivier Leservoisier, ed., Terrains ethnographiques et hiérarchies sociales. Retour réflexif sur la situation d'enquête », L'Homme [En ligne], 183 | 2007, mis en ligne le 28 juin 2007, consulté le 24 septembre 2020. URL : http://journals.openedition.org//homme/9651 ; DOI : https://doi.org/10.4000/ Ihomme.9651

Ce document a été généré automatiquement le 24 septembre 2020.

(c) École des hautes études en sciences sociales 


\title{
Olivier Leservoisier, ed., Terrains ethnographiques et hiérarchies sociales. Retour réflexif sur la situation d'enquête
}

Paris, Karthala, 2005, 327 p., bibl., index (« Hommes et sociétés »)

\author{
Samuel Lézé
}

1 La réflexivité est une exigence méthodologique et épistémologique qui reste marquée au sceau de l'ambiguïté. Si les anthropologues ne peuvent faire l'économie d'un retour critique sur les conditions de production de leur savoir, ni sur la construction de leur objet, ce n'est pas pour se complaire dans l'autocritique, l'exaltation de la « réflexivité narcissique » ou la glose littéraire des choix d'écriture qu'alimente une posture sceptique à l'égard du projet théorique de l'anthropologie. Loin d'être un renoncement, il s'agit au contraire de tirer parti des limites intrinsèques et même des sources d'erreur importantes du travail de terrain liées aux aléas de la relation d'enquête en tant qu'elle introduit un acteur particulier dans un champ social spécifique. En affrontant cette difficulté et les rectifications qu'elle implique, on améliore la connaissance de son objet, garantit l'objectivité des résultats et la scientificité de la démarche ${ }^{1}$.

Olivier Leservoisier exprime clairement son projet dans sa très judicieuse mise au point à propos de la réflexivité et de ses limites, du contexte intellectuel récent concernant les critiques de l'anthropologie et des paradoxes du travail de terrain trop longtemps considéré dans son évidence (pp. 5-19). La littérature anthropologique française ${ }^{2}$ est en effet longtemps demeurée extrêmement discrète sur les politiques du terrain excepté pour ceux qui furent très tôt sensibilisés aux terrains du politique grâce aux œuvres de Georges Balandier, Pierre Bourdieu, Gérard Althabe (certains articles rendent hommage à ce dernier, récemment disparu) ou aux travaux africanistes qui démontrent depuis longtemps déjà l'importance des situations sociales dans l'analyse de leur objet. En souhaitant penser ensemble ces deux registres, la démarche proposée ici est très originale : si, au sens premier, la réflexivité n'est rien d'autre qu'un travail de réduction 
de notre propre ethnocentrisme, sa visée épistémologique peut laisser place à une visée plus théorique lorsqu'elle devient également analyse de l'ethnocentrisme spontané dont font preuve tous groupes sociaux au travers de leur ordre social. Dès lors, il s'agit d'illustrer la richesse théorique de la réflexivité sur une problématique précise (les hiérarchies sociales) et sur des situations d'enquête spécifiques pour rompre avec toute forme de posture sceptique ou postmoderne.

3 Cinq objectifs majeurs fixent rigoureusement le cahier des charges qu'olivier Leservoisier donne à son entreprise : rendre compte de l'historicité d'une recherche, de la place de l'ethnologue dans un espace social donné, des changements affectant un terrain, puis tirer les enseignements méthodologiques et théoriques de l'étude des hiérarchies sociales et, enfin, souligner la portée heuristique de la réflexivité en anthropologie (p. 20). Une fois refermées les dernières pages de ce volume collectif, j'avoue avoir quasiment oublié l'intention initiale ; peut-être n'avais-je guère besoin d'être convaincu ou bien est-ce le signe de sa réussite, mais ce qui s'impose finalement à ma lecture, c'est une contribution d'anthropologie politique à l'analyse des classifications sociales et des enjeux de pouvoir à partir d'un retour réflexif sur la situation d'enquête (pp. 21-29). Au moyen de trois études, la première partie formule les grandes lignes que développeront les deux suivantes. Par la narration d'une enquête de terrain de longue durée (au Cameroun), Jean-Pierre Warnier décrit, non sans humour, le travail progressif et lent qui lui a permis d'élucider l'ancrage corporel (i.e. non discursif) des hiérarchies et une microphysique du pouvoir. Après un long processus d'intégration et d'observation (en Inde du Sud auprès des Intouchables), le texte sévère ${ }^{3}$ de Robert Deliège rompt avec les prénotions savantes à l'égard des hiérarchies en Inde et plus particulièrement de la place des Intouchables. L'analyse comparative de deux terrains (au Maroc et au Soudan) menée par Barbara Casciarri donne à voir toute la complexité des dimensions qui se croisent dans la situation d'enquête : l'inscription de l'anthropologue dans les hiérarchies (y compris sexuelle) et les transformations de son statut.

4 Une fois clarifiées les notions de base et enrichi le cadre analytique, la deuxième partie peut être entièrement consacrée à montrer la construction d'un objet d'investigation au cœur même des situations rencontrées lors du travail de terrain. Comme le dit Jeanne Favret-Saada, l'existence d'un système de positions n'apparaît que lorsqu'on s'y cogne ${ }^{4}$. Cinq articles illustrent très clairement le processus à l'œuvre : l'esclavage en Mauritanie en introduisant l'implication émotionnelle du chercheur (Olivier Leservoisier), les questions d'ethnicité en Colombie (Élisabeth Cuni), les enjeux d'un mariage arrangé au Mali (Gilles Holder \& Emmanuelle Olivier), les conséquences locales de la mondialisation sur une petite ville minière du nord de la France et sur une entreprise industrielle de Côte-d'Ivoire (Laurent Bazin), ou encore, le fonctionnement d'un laboratoire de biologie (Valéria A. Hernandez). À cet égard, si le rôle catalyseur de la réflexivité des "nouveaux terrains" de l'anthropologie est bien souligné dans la préface (pp. 14-16), ces deux derniers articles en sont les uniques témoins. Il s'agit là de mon seul regret.

5 Avec des arguments historiques et sociologiques, la dernière partie interroge les catégories d'analyse des hiérarchies sociales à l'aune des transformations sociales: l'article de Marie-Odile Géraud déconstruit ainsi l'image culturaliste enchantée de la société " égalitaire et homogène » hmong telle qu'elle s'actualise dans la communauté hmong de Guyane ; Jean Copans (la confrérie mouride au Sénégal) et Pierre Bonte (une 
entreprise minière de Mauritanie) proposent une analyse très suggestive de deux terrains "revisités » d'où surgissent les ressorts de la permanence et de la mémoire longue ; Serge Tcherkezoff introduit, pour sa part, un utile distinguo entre hiérarchie et stratification pour éclairer un malentendu longtemps entretenu sur son terrain (aux Samoa).

Il est en définitive possible de faire une « double lecture » de chaque contribution : une lecture théorique que l'on mesure à son apport à un domaine de recherche et une lecture méthodologique que l'on mesure surtout, si je m'en tiens à mon expérience d'enseignant, à son excellente exploitation pédagogique. Car pour être efficace, la réflexivité doit devenir collective, se généraliser à l'ensemble de la discipline (sous forme "d'anthropologie de l'anthropologie », p. 29) et se muer en habitus scientifique (une " réflexivité réflexe »). Or, elle ne peut certainement pas se transmettre comme un savoir, un ensemble de règles à appliquer. Comme un métier, la réflexivité s'apprend donc patiemment en faisant ou en voyant faire. Remplacer les leçons par le récit du processus de découverte est une proposition didactique déjà ancienne de Gaston Bachelard qui assumait à la fois le sens pédagogique et théorique de la formation de l'esprit scientifique: "puisqu'il n'y a pas de démarche objective sans la conscience d'une erreur intime et première, nous devons commencer les leçons d'objectivité par une véritable confession de nos fautes intellectuelles. Avouons donc nos sottises pour que notre frère y reconnaisse les siennes, et réclamons de lui l'aveu et le service réciproque $»^{5}$. La réflexivité est une pièce maitresse dans la formation de l'esprit anthropologique. C'est ce qu'établit l'ouvrage en réunissant douze situations d'enquête qui pourront servir à transmettre un habitus scientifique. À ce jour, il n'existe pas meilleur ouvrage de référence.

\section{NOTES}

1. Cf. Samuel Lézé, « Réflexivité », in Jean-Philippe Cazier, ed., Abécédaire de Pierre Bourdieu, Paris, J. Vrin/Mons, Sils Maria, 2007 : 159-161 ; Christian Ghasarian, « Les désarrois de l'ethnographe », L'Homme, 1997, 143 : 189-198.

2. Cf. Daniel Céfaï, L'Enquête de terrain, Paris, La Découverte, 2003, et mon compte rendu de cet ouvrage dans L'Homme, 2005, 175-176 : 487-490.

3. Suspicieux à l'égard des « nouveaux terrains » et de la mode réflexive, il assume volontiers (pp. 52-53) une vision old-fashioned ou orthodoxe de l'anthropologie sociale professée naguère à Oxford et qui exigerait « une réaffirmation forte de ses objets et de ses méthodes »... Évidemment, concède-t-il, l'ethnologie est « capable de tolérer des manifestations atypiques "...

4. Voir l'entretien de Jeanne Favret-Saada, «Glissements de terrains », Vacarmes, 2004, 28 (http://www.vacarme.eu.org/article449.html).

5. Gaston Bachelard, La Formation de l'esprit scientifique, Paris, Vrin, 1970 : 242-243. 


\section{AUTEUR}

\section{SAMUEL LÉZÉ}

ENS, Laboratoire de sciences sociales, Paris.

Samuel.Leze@ens.fr 